\title{
EDITORIAL ROLE OF LEE SILVERMAN VOICE TREATMENT
(LSVT) IN PARKINSON DISEASE
}

Parkinson Disease is a progressive neurological disorder that affects approximately seven to ten million population across the globe, prevalence of disease increase with age, nearly four percent of total population suffering from Parkinson Disease are diagnosed before the age of 50 years, men are having one and a half times more chance of developing a disease as compared to females. It is the sixth most common among the neurological disorders seen in urban United Kingdom, with an incidence of about $2.8 \%$ in the US; unfortunately there is lack of related statistics available for Pakistani population. However, there is a reported increase in the overall prevalence of neurological diseases in Pakistan and it is reported that more middle aged Pakistani males suffers from Parkinson Disease as compared to females, displaying a worsening of symptoms and possible deficits in cognition as age progresses.

Parkinson disease (PD) mainly impairs limb motor functions, gait, balance, swallowing, speech and activities of daily living of individuals. Optimal medical care inclusive of pharmacological and surgical management have not been able to control these deficits to a satisfactory level in majority of patients with PD and nearly all of them have suffered a negative impact on quality of their life at one stage or another. It is mandatory to understand neural mechanisms of both movement and speech disorders in Parkinson Disease in order to incorporate a better treatment mechanism that may improves treatment outcomes and ultimately improving health related quality of life in such patients.

Neuroplasticity is the ability of brain to bring change in neural pathways and synapses by engaging throughout life in synaptic pruning and deleting no longer required or useful neural connections and in addition strengthening those that are needed now due to certain environmental, neural or behavioral factors. Lee Silverman Voice Treatment (LSVT), a neuro-plasticity treatment approach is a recent exercise -based behavioral treatment program used in individuals suffering from Parkinson disease and other neurological disorders like stroke, multiple sclerosis and in children like cerebral palsy and Down syndrome.

LSVT (Lee Silverman Voice Treatment) Programs has been developed and researched over the period of 20 years. This program, rather only relying on retaining deficient functions, focuses on combined approach of 1), increasing amplitude of motor system in both speech and gross movements in the limb motor system, 2), sensory recalibration to assist patients in recognizing that increase amplitude of movements are within normal limits, and 3), also training self-cueing and being more aware of the actions taken to facilitate continuation of treatment outcomes for longer period of time. It is an intensive delivery model that focuses on principle of activity-dependent neuroplasticity and motor learning. It has two main focuses one on speech motor system (LSVT LOUD) and more recently it has also extended its focus to address deficiencies in limb motor systems (LSVT BIG). Advances in neuro-rehabilitation are advocating the role of the said protocol in improvement of functions and possible slow progression of motor symptoms in patients with Parkinson disease. It can be delivered by speech and language therapist, physical therapist and occupational therapist according to the desired outcomes.

Preliminary studies have documented LSVT as an effective tool in overall improvement of quality of life, amplitude of limbs and body movement, ability to deal with more than one task at a time, improves articulation, facial expression, and swallowing in patients suffering from the disease. LSVT has been advocated as unique, complex and intensive protocol that ensures optimized learning by high effort exercises to specifically address movement impairments in Parkinson disease. Majority of researches conducted on LSVT-LOUD are case studies or small group designs on individuals with idiopathic Parkinson Disease, results of LSVT outcomes are yet to be documented on Randomized control trails and on individuals suffering from these deficits after neurosurgery and with atypical Parkinson. Future studies would also be required to better define prognostic variables in response to LSVT Programs and how expected outcomes can be altered by the severity of symptoms and by the presence of factors like dementia, depression, and apathy in idiopathic PD, atypical PD and post-Deep Brain Stimulation surgical procedures as the magnitude of outcome may not be same as has been observed till date. These studies will help address the underlying bases for treatment-related changes that would impact better on speech and movement and thus be beneficial in improving quality of life in individuals with Parkinson disease. 
Pakistan is a third world country where $39 \%$ of its population living below the poverty line $19.3 \%$ in urban areas and $54.6 \%$ in rural areas). There is lack of primary health care in rural areas with "14.5 physicians per 10,000 population in urban areas," as compared to "3.6 per 10000 population in rural areas,". With the government spending a meager amount on primary health care, early intervention of illness or disease is unlikely in most cases. An increase in longevity with a severe compromise in quality of life is reported by Ikram Junidi because of an increase in non-fatal illnesses and injuries, having varied origin such as neurological, musculoskeletal, substance abuse and even mental disorders. This warrants a tremendous need for rehabilitation of this population of patients.

Early referral for rehabilitation along with medication in patients diagnosed with neurological disorders like Parkinson disease can contribute immensely in the alleviation of most of the symptoms of disease and for improving and or maintaining better quality of life. Basically, early intervention is key to successful rehabilitation of any patient and more so in neurological disorders. Globally, Last two decades have witnessed drastic changes in approach to Neuro-Rehabilitation not only due to the introduction of modern exercise protocols, their easy accessibility to patients through computer and web based technology, advancement of technology like computerized robotic electronic devices such as robotic lower limb orthoses, upper limb training robots, transcranial magnetic stimulation and neuro prosthesis. All these regimes are designed to produce positive changes in cortical excitability of the damaged cerebral hemisphere thus improving neuroplasticity and ultimately quality of life in such patients. It is the need of time that Pakistan health sector should also entre the era of this growth to alleviate the suffering of our neurologically compromised society.

\section{Sumaira Imran Farooqui}

Associate Professor

Ziauddin College of Physical Therapy

Ziauddin University 